\title{
Critical Residues of $\mathrm{G} \beta \gamma$ for the interaction with the SNARE Complex
}

Benjamin K. Mueller ${ }^{1}$, Ali I Kaya ${ }^{2 \dagger}$, Zack Zurawski ${ }^{2 \dagger \dagger}$, Yun Young Yim ${ }^{2 \dagger \dagger}$, Jens Meiler ${ }^{1,3}$, Heidi E. Hamm ${ }^{2}$

${ }^{1}$ Department of Chemistry, Vanderbilt University, 7330 Stevenson Center, Station B 351822, Nashville, Tennessee 37235, USA

${ }^{2}$ Department of Pharmacology, Vanderbilt University, Nashville, Tennessee 37232-6600, USA

${ }^{3}$ Institute for Drug Development, Leipzig University, Leipzig, Germany

${ }^{\dagger}$ Currently, NE-CAT, Department of Chemistry and Chemical Biology, Cornell University, Argonne National Laboratory, Argonne, IL 60439, USA

${ }^{\dagger}$ Currently, Department of Anatomy and Cell Biology, University of Illinois at Chicago, Chicago, IL 60612, USA

${ }^{\dagger \dagger}$ Currently, Nash Department of Neuroscience, Icahn School of Medicine at Mount Sinai, New York, NY 10029, USA

Correspondence: Heidi E. Hamm, heidi.hamm@vanderbilt.edu 


\begin{abstract}
The mechanisms and regulation of neurotransmitter release is a complex process involving many co-factors and proteins. One critical interaction is the regulation of exocytosis when Gprotein $\beta \gamma(\mathrm{G} \beta \gamma)$ dimers bind to the soluble N-ethylmaleimide-sensitive factor attachment protein receptor (SNARE) protein complex. The complex is comprised of N-ethylmaleimide-sensitive factor attachment protein-25 (SNAP-25), syntaxin 1A, and synaptobrevin. Herein we probe across the entire family of human $\mathrm{G} \beta$ and $\mathrm{G} \gamma$ proteins for residues critical for the interaction with SNARE, by systematically screening peptide sequences for their ability to bind to tSNARE. The coiled-coil region of G $\beta \gamma$ showed high affinity to tSNARE, along with the propeller region of $G \beta$ on the opposite side from the coiled-coil region. Peptides based on $\mathrm{G} \beta_{1} \gamma_{2}$, shown to have high affinity to SNARE, tSNARE were screened further by alanine scanning to probe for residues critical for binding to tSNARE. Full length $\mathrm{G} \beta_{1} \gamma_{2}$ and SNARE were docked computationally using Rosetta, to examine the experimentally determined binding sites. Docking converged on two possible sites of interaction using two distinct regions of both $G \beta_{1} \gamma_{2}$ and SNARE.
\end{abstract}

\title{
Introduction
}

Exocytosis is a complex, regulated process involving the exocytotic machinery proteins, synaptic proteins that play roles in docking and priming the vesicle, ion channels, calcium sensors, and pre-synaptic inhibitory G-protein coupled receptors (GPCRs). Despite numerous attempts to understand the synaptic molecular composition and function, the microarchitecture of exocytosis with various modulators and synaptic proteins at each step has still remained unclear.

G $\beta \gamma$ subunits mediate fast, membrane-delimited effects on transmission, through both postsynaptic activation of GIRK channels (Logothetis et al., 1987; Reuveny et al., 1994) and 
binding voltage-gated calcium channels (VGCC) and modulation of calcium entry (Herlitze, 1996; Ikeda, 1996). At presynaptic terminals, $G \beta \gamma$ is an important regulator of neurotransmission through interactions with calcium channels (Herlitze, 1996; Ikeda, 1996) and via it's interaction with the SNARE complex which directly inhibits exocytosis (Betke et al., 2012; Blackmer et al., 2005; Blackmer et al., 2001; Gerachshenko et al., 2005). The interaction of G $\beta \gamma$ with the SNARE complex places inhibitory regulation of exocytosis at the final step of exocytotic fusion (Wells et al., 2012b).

There are five different G $\beta$ and twelve different $G \gamma$ subunits (Downes and Gautam, 1999; Hildebrandt, 1997; Simon et al., 1991). G $\beta$ subunits are made up of an $\alpha$-helix of approximately 20 amino acids and 7 blades of four anti-parallel $\beta$-strands, a propeller like WD repeat (Clapham and Neer, 1997; Gautam et al., 1998; Smrcka, 2008; Sondek et al., 1996b). G $\beta 1-4$ share up to $90 \%$ amino acid sequence identity whereas $G \beta_{5}$ is only $50 \%$ identical (Betty et al., 1998; Smrcka, 2008). In contrast, G $\gamma$ subunits share only $30-70 \%$ sequence identity (Betty et al., 1998; Smrcka, 2008). Made up of two $\alpha$-helices, the C-terminal $\alpha$ helix interacts with the surface of blade 5 and a small section of the $\mathrm{N}$ - terminal region on G $\beta$ while the N-terminal $\alpha$ helix forms a coiled-coil interaction with the N-terminal helix of the $\beta$ subunit (Clapham and Neer, 1997). G $\gamma$ subunits can be post-translationally modified at the processed C-terminal cysteine which is carboxymethylated and modified with a farnesyl or geranylgeranyl moiety via a thioether bond. These modifications mediate membrane localization of G $\beta \gamma$ (Clarke, 1992; Cox, 1995). Together, G $\beta$ and G $\gamma$ subunits form G $\beta \gamma$ dimers, and once assembled, act as signaling units for GPCRs and modulate synaptic transmission by interacting with a number of effectors such as voltage-gated calcium (VGCC) and inward-rectifying potassium (GIRK) channels, and soluble NSF attachment protein receptors (SNARE) to regulate neurotransmitter 
release at the synapse (Blackmer et al., 2001; Currie, 2010; Gerachshenko et al., 2005; Herlitze et al., 1996; Huang et al., 1995; Sadja and Reuveny, 2009; Wells et al., 2012a; Yoon et al., 2007).

SNAP25 is the most important G $\beta \gamma$ partner in the interaction between G $\beta \gamma$ and SNARE, a trimer of SNAP25, syntaxin 1A, and synaptobrevin (VAMP) and in the interaction between G $\beta \gamma$ and the tSNARE dimer, SNAP25 with syntaxin 1A (Blackmer et al., 2005; Blackmer et al., 2001; Delaney et al., 2007; Gerachshenko et al., 2005; Photowala et al., 2006; Yoon et al., 2007; Yoon et al., 2008; Zhang et al., 2011; Zhao et al., 2010). Multiple studies have shown the carboxy(C)terminus of SNAP25 to be important for the exocytotic function and its interaction with G $\beta \gamma$ botulinum toxin A (BoNT/A) cleaves the C-terminus of SNAP25 reduces G $\beta \gamma / \mathrm{SNARE}$ binding, and a 14-amino acid peptide of the C-terminus of SNAP25 inhibits the formation of the

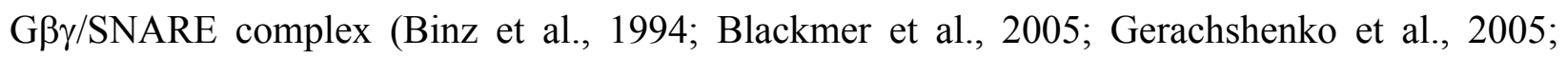
Schiavo et al., 1993; Yoon et al., 2007; Zhao et al., 2010). In Wells et al, in vitro binding studies have demonstrated direct G $\beta \gamma$-SNARE interaction sites at both the vesicle proximal C-termus as well as the other end of the SNARE complex where zipping up begins, as well as in the $\mathrm{H}_{3}$ domain of syntaxin (Wells et al., 2012a; Yoon et al., 2007). In addition, Asp99 and Lys102 in the linker region between the two helices of SNAP25 in close proximity to its palmitoylation sites (Cys 85, 88, 90, and 92) may be important for this interaction. Interestingly, Gly63 and Met64 that had significant reduction in binding are buried in the interface with syntaxin 1A and VAMP.

Despite numerous studies probing the sites on the SNARE complex of G $\beta \gamma$ interaction, it is still unclear where on G $\beta \gamma$ the interaction sites are. The G $\beta \gamma$-effector interaction sites were hypothesized to be localized at the Ga interface (Ford et al., 1998). However, mutagenesis studies revealed the binding regions of effectors within the blades of the G $\beta$ propeller and its N.terminus 
coiled.coil, the G $\alpha$ subunit interface, and regions on G $\gamma$ subunit (Clapham and Neer, 1997; Myung et al., 2006; Panchenko et al., 1998; Peng et al., 2003; Smrcka, 2008). For example, GIRK channels were found to interact just outside of the G $\alpha$ binding site. Thr 86, Thr87, and

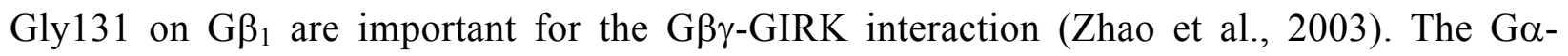
interacting residues that were shown to be important for G $\beta \gamma$ interaction with GIRK, VGCC, PLC $\beta$, GRK2, and G $\alpha$ (Ford et al., 1998) were shown to actually be inhibitory for interaction with the SNARE complex and inhibition of exocytosis; removal of those residues and replacement with Ala actually increases their affinity (Blackmer et al., 2005). Mutation of both Lys78 and Trp332 on G $\beta_{1} \gamma_{2}$ to Ala increased the affinity of G $\beta_{1} \gamma_{2}$ for tSNARE by two-fold (Zurawski et al., 2017b). While the interface of G $\alpha$ subunits likely represents a core site of G $\beta \gamma$ and its effector binding, other regions may also play important roles in facilitating G $\beta \gamma$ mediated downstream signaling.

We have recently reported specificity of $G \beta \gamma$ interaction with $\alpha_{2 \mathrm{a}} \mathrm{AR}$ and the SNARE complex based on proteomic studies (Yim et al., 2019) (Yim et al., 2020). Here, we address the structural basis of the specificity of different G $\beta$ and $G \gamma$ subunits for the SNARE complex using the method of peptide arrays (Yim et al., 2015a) to further understand the G $\beta \gamma$-SNARE interaction. The interaction is probed further by computationally docking G $\beta \gamma$ to SNARE, and evaluating the models against the mutagenesis screens.

\section{Results}

To characterize the tSNARE binding sites on G protein $\beta$ and $\gamma$ subunits, we used a peptide array assay. Peptides from across the sequences of human $\mathrm{G} \beta$ and $\mathrm{G} \gamma$ protein were synthesized by using Respep SL as described (Yim et al., 2015b). The spots on nylon membrane consisted of 15-mer 
peptides shifting three amino acids for each successive peptide which cumulatively represents the entire sequence of indicated proteins. Fig 1 A shows a representative blot and the average data for the interaction between $G \beta_{1}$ and tSNARE. The data revealed 6 major regions of $G \beta_{1}$ that make up the interaction with tSNARE protein. When we tested other G $\beta$ subunits, we found similar binding pattern between $\mathrm{G} \beta_{1-4}$ and tSNARE (Fig 1B). G $\beta_{5}$ showed low binding affinity compare to others. This binding data suggests that $\mathrm{G} \beta_{1-4}$ has multiple interacting sites with tSNARE protein (Fig 1C).

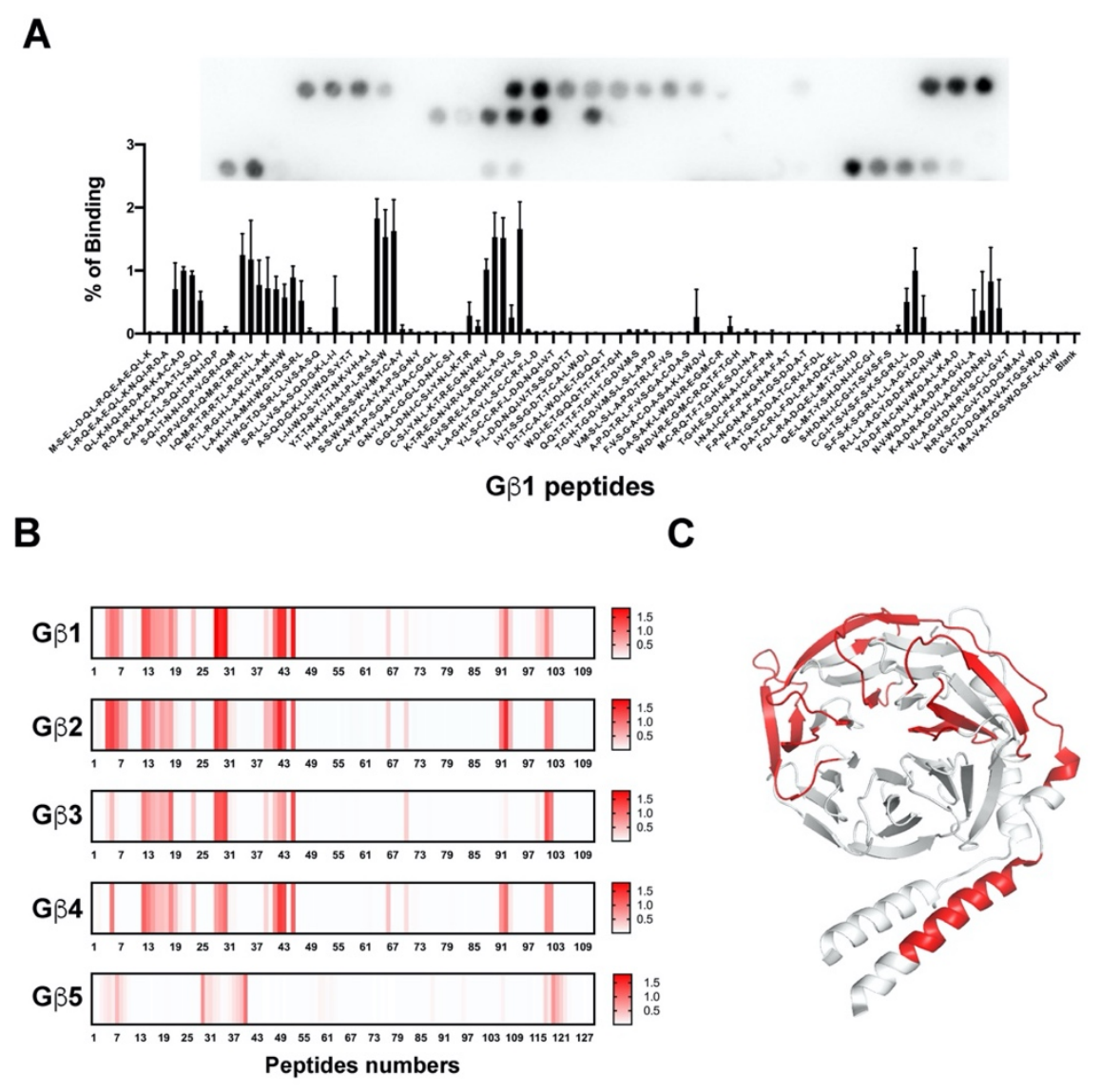


Fig 1 tSNARE binding sites on G $\boldsymbol{\beta}$ subunits. A) Representative peptide blot (15-mer) of human G $\beta 1$ and quantified binding of tSNARE to G $\beta_{1}$ peptides. Binding was measured as a percentage of total intensity on the membrane. A 15-mer glycine peptide was used to determine non-specific interactions. Human SNAP25 peptides between amino acids 91105 and 106-121 were used as positive control for SNAP25 antibody B) Heat map using a single gradient to display ranges of binding between tSNARE and G $\beta_{1-5}$ was calculated using GraphPad Prism 8.0.2. C) Map of interaction sites for tSNARE to $\mathrm{G}_{1}$ are highlighted in red (PDB 1GP2(Wall et al., 1995)).

We used the same approach to detect interacting regions of G $\gamma$ with tSNARE. Figure 2A shows binding between $\mathrm{G} \gamma_{1}$ and $\mathrm{G} \gamma_{2}$ and tSNARE. The data clearly indicate that the binding is significantly higher with $\mathrm{G} \gamma_{2}$ peptides than $\mathrm{G} \gamma_{1}$ (Fig. 2A). It appears that there are two major binding sites between $\mathrm{G} \gamma_{2}$ and tSNARE, one at the N-terminus and the other at the C-terminus of $\mathrm{G} \gamma_{2}$ (Fig. 2B). This is consistent with our previous study(Zurawski et al., 2017a). When we investigated the other $\mathrm{G} \gamma$ subtypes, we observed similar binding patterns as $\mathrm{G} \gamma_{2}$ (Fig. 2C). This result indicates that $\mathrm{G} \gamma_{1}$ has lower affinity to tSNARE, and suggests that the other $\mathrm{G} \gamma$ subtypes have two binding sites at the $\mathrm{N}$ - and the $\mathrm{C}$-termini of the protein. 

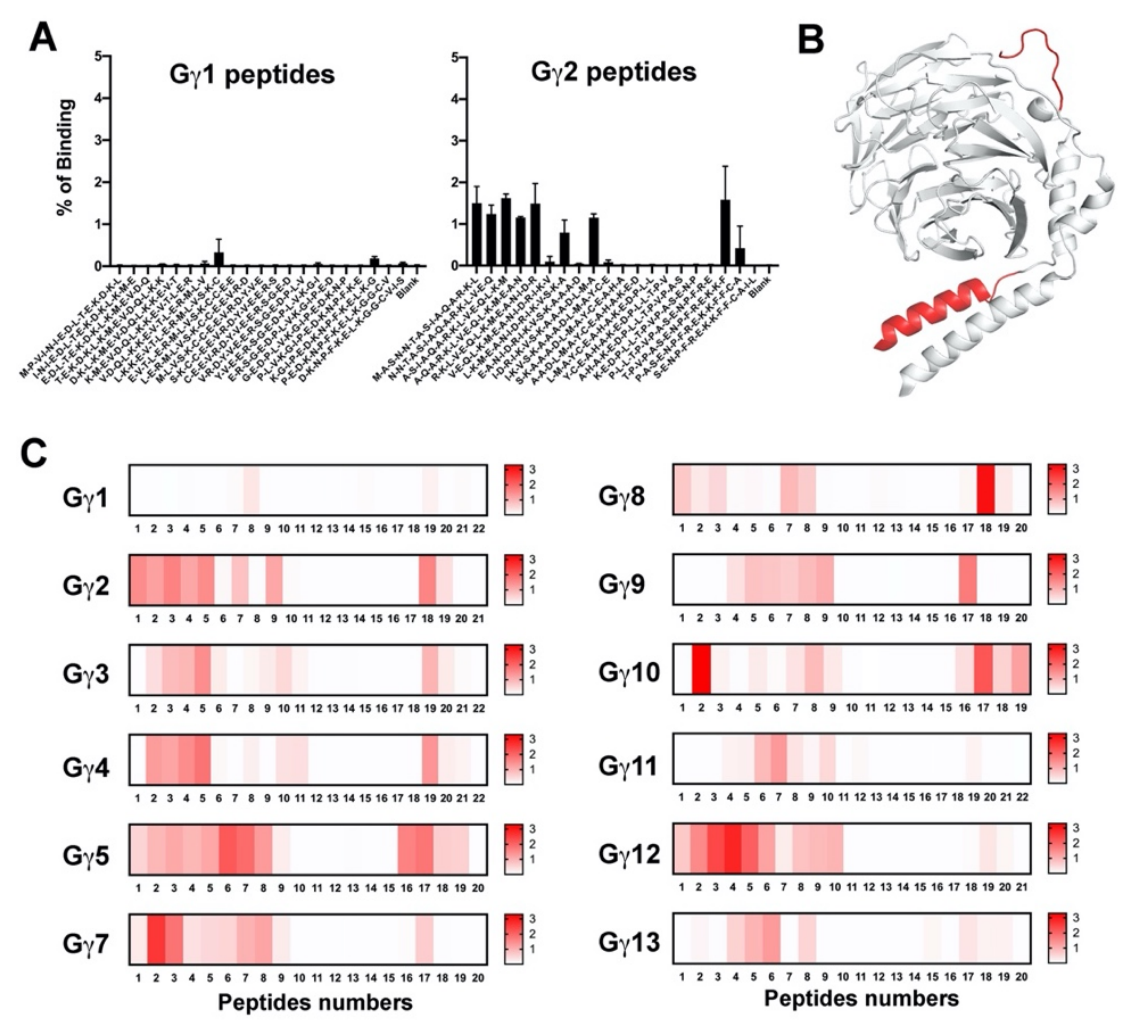

Fig 2 tSNARE binding sites on G $\gamma$ subunits. A) Representative quantified binding of tSNARE to $\mathrm{G} \gamma_{1}$ and $\mathrm{G} \gamma_{2}$ peptides. Binding was measured as a percentage of total intensity on the membrane. A 15-mer glycine peptide was used to determine non-specific interactions. B) Map of interaction sites for tSNARE to $\mathrm{G} \gamma_{2}$ are highlighted in red (PDB 1GP2 (Wall et al., 1995)). C) Heat map using a single gradient to display ranges of binding between tSNARE and $\mathrm{G} \gamma_{1-13}$ was calculated using GraphPad Prism 8.0.2.

On the basis of these results, selected peptides that bound tSNARE in this screen were then subjected to alanine-scanning mutagenesis. For each peptide, the first spot was the wild-type sequence of a given peptide followed by spots with a single alanine mutation. Fig 3A shows alanine scanning from $G \beta_{1}$ and $G \gamma_{2}$ peptides. The bar graphs demonstrates the average densitometry of all peptides with loss (or gain) of binding in a mutant. The result indicate that positively charged residues play a key role for both $\mathrm{G} \beta_{1}$ and $\mathrm{G} \gamma_{2}$ interaction with tSNARE protein. 
Fig 3B shows the location of the peptides (green) and important residues (blue) for tSNARE interaction.

A
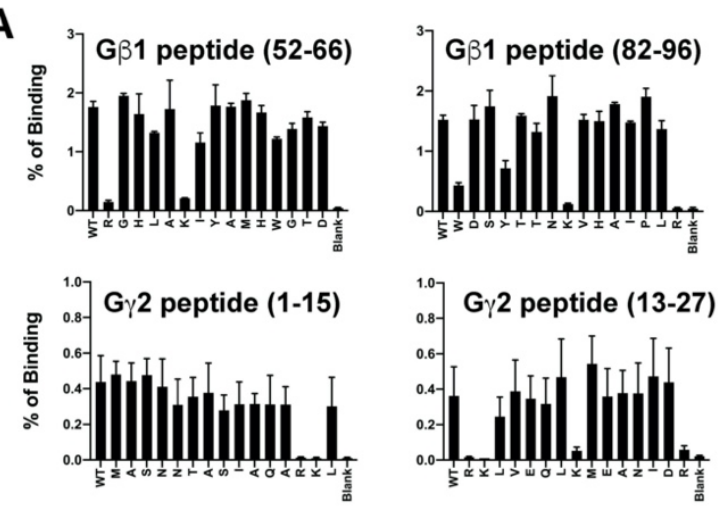
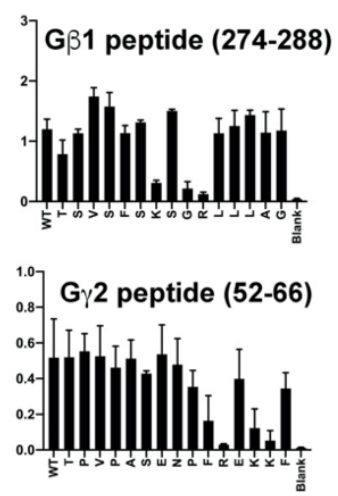

B
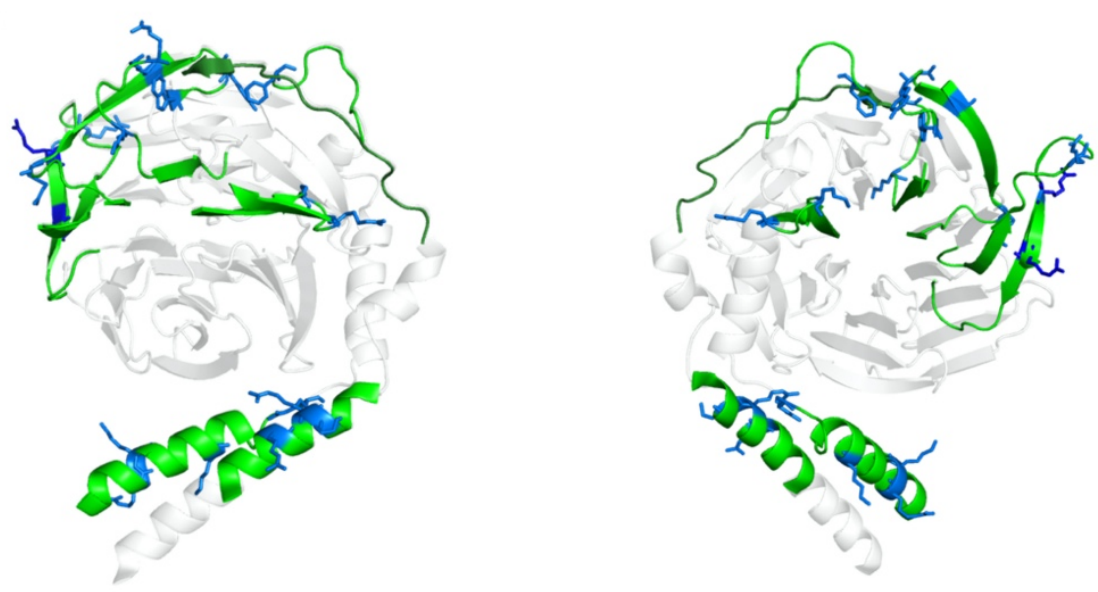

Fig 3 Alanine mutagenesis screening of G $\beta \gamma$ peptides that bind tSNARE. A) Selected peptide regions from $\mathrm{G} \beta_{1}$ and $\mathrm{G} \gamma_{2}$ subunits were mutated to alanine. The first bar of each graph contains wild-type peptide. The next 15 bars of each graph to the right are mutant peptides with a single alanine replacement of the residue at position 1,2,3.., 15 for each wild-type peptide. B) The peptides are mapped onto 1GP2 structure G $\beta \gamma$ subunit and shown in green. The residues that had significantly reduced binding between G $\beta \gamma$ with tSNARE are shown with blue (stick).

Previous studies showed that the affinity of $G \beta_{1} \gamma_{1}$ is much lower than $G \beta_{1} \gamma_{2}$ for both monomeric SNAP25, t-SNARE, and the ternary SNARE complex. Since the only difference is within the G $\gamma$ 
subunit between these two G protein subtypes, we decided to investigate the affinity of different G $\gamma$ subunits to SNAP25. We measured the ability of peptides to disrupt the interaction between full-length G $\beta_{1} \gamma_{2}$ with SNAP25 using the Alphascreen competition-binding assay (Zurawski et al., 2017a). Fig 4 shows the IC50 differences between $G \gamma_{1}$ and $G \gamma_{2}$. This result is consistent with previous studies (Zurawski et al., 2017a). We also tested other G $\gamma$ subtype peptides (Table 1) and the data indicate that $\mathrm{G} \gamma$ subtypes showing varying affinities for SNAP25. For instance, G $\gamma_{2}$ and $\mathrm{G} \gamma_{10} \mathrm{~N}$-terminus and $\mathrm{G} \gamma_{8} \mathrm{C}$-terminus has higher affinity for SNAP25 compared to other peptides.

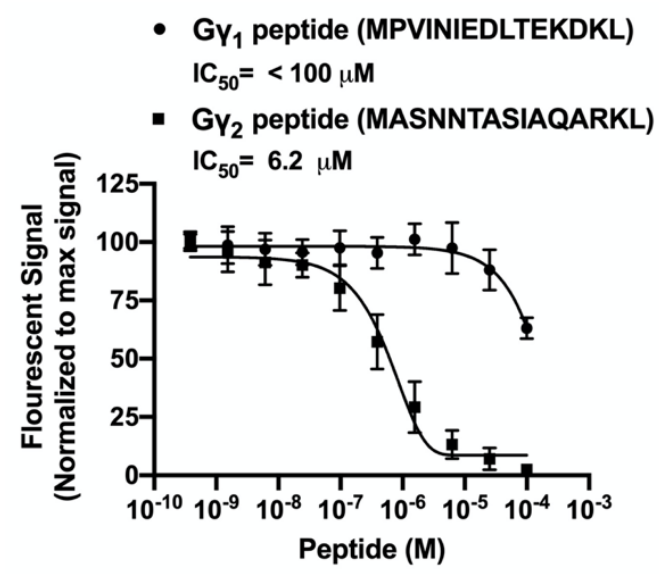

Fig 4 Competition assays of $G \beta \gamma$-derived peptides to inhibit G $\beta_{1} \gamma_{2} / \mathrm{SNAP}-25$ complex formation. Alphascreen competition-binding assays in which $20 \mathrm{nM}$ biotinylated SNAP25 reacts with $180 \mathrm{nM} \mathrm{G} \beta_{1} \gamma_{2}$ to produce a luminescent signal were conducted in the presence of peptides corresponding to primary sequences within $\mathrm{G} \gamma 1$ and $\mathrm{G} \gamma 2$ peptides at varying concentrations (1.08 $\mathrm{nM}$ to $100 \mu \mathrm{M})$ dissolved in DMSO. A value of $100 \%$ was assigned to the average of all conditions tested containing only DMSO as a positive control. The primary sequence of each peptide is depicted for each condition. Experiments were repeated three times. IC $_{50}$ values and 95\% confidence intervals were observed. IC50 of the selected $\mathrm{G} \gamma$ peptides are shown in Table 1. 


\begin{tabular}{|ccc|}
\hline G gamma & Sequence & IC50 $(\boldsymbol{\mu M})$ \\
\hline Gy1 & MPVINIEDLTEKDKL & $<100$ \\
\hline Gy2 & MASNNTASIAQARKL & 6.2 \\
\hline Gy2 & TPVPASENPFREKKF & $<100$ \\
\hline Gy3 & IGQARKMVEQLKIEA & 62.4 \\
\hline Gy3 & ITPVPTSENPFREKK & 82.5 \\
\hline Gy5 & LTGSSSTNPFRPQK & 42.3 \\
\hline Gy8 & PVPAAENPFRDKRLF & 10.6 \\
\hline Gy10 & GAGASALQRLVEQLK & 5.25 \\
\hline Gy12 & STNNIAQARRTVQQL & 60.5 \\
\hline Gy13 & LKYQLAFOREMASKT & $<100$ \\
\hline
\end{tabular}

Table 1 Alphascreen competition-binding assays. Competition assays between biotinylated SNAP25 and G $\beta_{1} \gamma_{2}$ were conducted in the presence of indicated $\mathrm{G} \gamma$ peptides.

The interaction between $\mathrm{G} \beta_{1} \gamma_{2}$ and SNARE was further probed via computational docking. The structures of SNARE(Ernst and Brunger, 2003) and $\mathrm{G}_{1} \gamma_{2}$ (Sondek et al., 1996a) have been determined alone and with other protein binding partners (Chen et al., 2002; Huang et al., 2019; Lambright et al., 1996; Maeda et al., 2018), and have not exhibited large conformational changes. Therefore the crystal structures of SNARE and G $\beta_{1} \gamma_{2}$ using the ROSETTADOCK (Chaudhury et al., 2011; Gray et al., 2003) protocol of ROSETTA (Bender et al., 2016), as guided by the interaction sites identified by peptide array analysis and mutagenesis. Two regions of the G $\beta_{1} \gamma_{2}$ were probed - the coiled-coil region and the propeller region on the far side of $G \beta_{1}$ away from the coiled-coil. These regions were docked with the C-terminus of SNAP-25 and near residues 151164 of SNAP-25, both found to be sensitive to mutation for complex formation.

The generated models were evaluated by the total energy of the complex and interface energy between the two binding partners (Fig 5). The lowest interface energy models were used as a point of reference and the root mean square deviation (RMSD) was calculated to these models (Fig 5a) to determine the convergence of the independent docking runs. 
These docking studies identify two distinct docking modes with good convergence. In the first binding mode, the N-terminus of G $\gamma$ binds between the two chains of SNAP25 at the C-terminus of the SNARE complex (Fig 5b). This model is consistent with the mutagenesis and prior screens. Our previous work (Zurawski et al., 2016) identified SNAP-25 $5^{\mathrm{R} 198}$ as a critical residue for the formation of the G $\beta \gamma$-SNARE complex. In the computational docking model, SNAP- $25^{\mathrm{R} 198}$ forms a salt bridge with $G \beta^{\mathrm{E} 3}$. In addition, the peptide mutagenesis of the $\mathrm{G} \gamma$ subunit suggested that the $\mathrm{G} \gamma$ residues R13/K14/K20 impact the interaction with SNARE, and these are all located at the proposed interface in the docking model (Fig 5c). This model predicts the parallel (as opposed to head-to-tail) orientation of the interactions, with the helical region of $\mathrm{G} \gamma$ lying flush with the SNARE coiled-coil. The computational model allows for complexin to bind SNARE and 


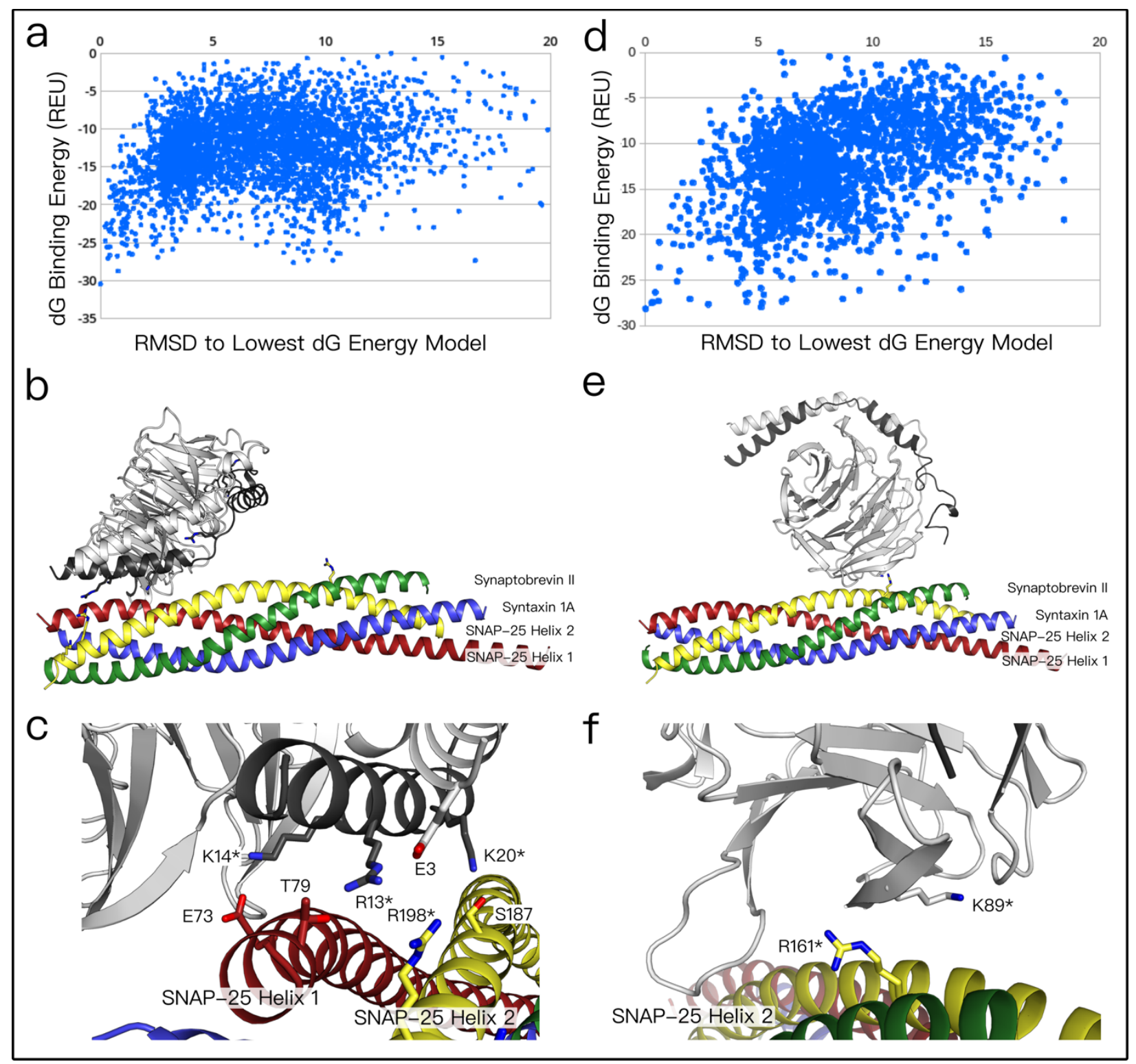


Fig 5 Computational docking of Gß $\gamma$ to SNARE. A) Plot of the $\Delta \mathrm{G}$ binding energy versus $\mathrm{C} \alpha$ RMSD to lowest energy model of SNARE/G $\beta \gamma$ complex at site 1. The structure of G $\beta \gamma$ (pdbid: 6crk) was computationally docked to the structure of SNARE (pdbid:1n7s) using RosettaDock. The lowest energy model, by binding energy, is shown in (B). B) G $\beta \gamma /$ SNARE complex lowest energy model at site 1 , by dG binding energy. Complex colored as: G $\beta$ - white, G $\gamma$ - black, green - Synaptobrevin II, blue - Syntaxin 1A, red - SNAP-25 (helix 1), yellow SNAP-25 (helix 2). Residues shown as sticks in the model were shown in to be sensitive to mutagenesis. C) Interface of model in (B) - the lowest energy model places the residues shown to be critical on $\mathrm{G} \gamma(\mathrm{R} 13, \mathrm{~K} 14, \mathrm{~K} 20$ and R27) by the interface of the interaction. R198, on helix 2 of SNAP-25, shown to be sensitive to mutagenesis (starred in fig). D) Plot of the $\Delta G$ binding energy versus C $\alpha$ RMSD to lowest energy model of SNARE/G $\beta \gamma$ complex at site 2 . E) GBy/SNARE complex lowest energy model at site 2, same coloring as (B). Residues shown as sticks in the model were shown in to be sensitive to mutagenesis. F) Interface of model in (E) - the lowest energy model places a residue shown to be critical on $G \beta, K 89$, by the interface of the interaction. R161, on helix 2 of SNAP-25, shown to be sensitive to mutagenesis (starred in fig).

G $\beta \gamma$ in a tripartite structure as in the SNARE-complexin structure (Chen et al., 2002).

A second, distinct, docking binding mode that is also consistent with the peptide array analysis and mutagenesis screens, places the G $\beta \gamma$ near the middle of the SNARE complex. In this model, the G $\beta^{82-96}$ and $G \beta^{124-138}$ of the propeller region were located adjacent to SNAP25 residues 151-164, and the critical residue R161. The models with the best convergence place SNAP-25 R161 adjacent to G $\beta$ K89, which was sensitive to alanine mutagenesis. While the residue side chains do not interact in either of the lowest energy models, the positively charged arginine side chain interacts with the backbone of residue 89 or 90 . 


\section{Discussion}

In general, $\mathrm{G} \gamma$ subunits may be classified as $\mathrm{G} \gamma_{1}$-like or $\mathrm{G} \gamma_{2}$-like. The first category consists exclusively of $\mathrm{G} \gamma_{1}$ and $\mathrm{G} \gamma_{11}$, while the ten other $\mathrm{G} \gamma$ subunits comprise the second category. A critical point of differentiation is found in the CaaX-box motif in each protein: the residue $\mathrm{X}$ is a serine in $\mathrm{G} \gamma_{1}$-like subunits, enabling farnesyltransferase to farnesylate the C-terminus. $\mathrm{G} \gamma_{2}$-like subunits instead have a leucine residue in this position, conferring geranylgeranylation from geranylgeranyltransferase I instead. It has been previously determined that $\mathrm{G} \gamma_{2}$-containing G $\beta \gamma$ subunits have substantially higher affinity for the SNARE complex and ability to inhibit exocytosis than $\mathrm{G} \gamma_{1}$-containing $\mathrm{G} \beta \gamma$ subunits (Blackmer et al, 2005, Yoon et al, 2007, Zurawski et al, 2017). Here, we derive new mechanistic insights into this difference in potency. First, we recapitulate the results from our previous peptide inhibition study in which peptides derived from the $\mathrm{N}$-terminus of $\mathrm{G} \gamma_{2}$ potently inhibit the binding of $\mathrm{G} \beta_{1} \gamma_{2}$ to monomeric SNAP25, whereas those derived from the N-terminus of $\mathrm{G} \gamma_{1}$ do not. We expand upon those studies with a range of potencies for peptides corresponding to different primary sequences within the family of $\mathrm{G} \gamma_{2}$-like subunits, with $\mathrm{N}$ terminal sequences within $\mathrm{G} \gamma_{3}$ and $\mathrm{G} \gamma_{12}$ providing minimal inhibition, while $\mathrm{G} \gamma_{2}, \mathrm{G} \gamma_{8}$, and $\mathrm{G} \gamma_{10}$ are six to sixteen-fold more potent at inhibiting the binding of $\mathrm{G} \beta_{1} \gamma_{2}$ to monomeric SNAP25. From this, we conclude that the primary sequence of the $\mathrm{G} \gamma$ subunit is contributory towards the binding interface of G $\beta \gamma$ with SNARE, rejecting the notion that the identity of the prenyl group attached to the $\mathrm{G} \gamma$ is the sole determinant of potency, perhaps due to more efficient membrane targeting.

Scanning alanine mutagenesis of the $\mathrm{G} \gamma_{2} \mathrm{~N}$-terminal peptide suggests that residue R13 and K14 are critical for binding to t-SNARE, as the interaction is abolished when these residues are mutated to Ala (Fig. 3). Alignment of primary sequences within the group of $\mathrm{G} \gamma_{2}$-like subunits using Clustal Omega shows a high degree of conservation of these key residues (Fig. 6) between 
each $\mathrm{G} \gamma$ subunit. Intriguingly, the most potent $\mathrm{N}$-terminal peptide derived from the primary sequence of $\mathrm{G} \gamma_{10}$, was the least conserved by far, featuring a weakly negatively-charged Gln instead of the positively-charged $\mathrm{R}$ and $\mathrm{K}$ residues. However, this may be consistent with previously published binding data, in which seven of nine G $\beta \gamma$-binding residues on SNAP25 are R or K residues (Wells et al, 2012). This may imply that $\mathrm{G} \gamma_{10}$ interacts with SNARE via structurally unique residues compared to other G $\beta \gamma$ heterodimers, since its interaction is predicted from these studies.

$$
\begin{aligned}
& \text { GY12 } \\
& \text { Gy8 } \\
& \text { Gү2 } \\
& \text { Gy3 } \\
& \text { GY10 } \\
& \text { Gү5 }
\end{aligned}
$$

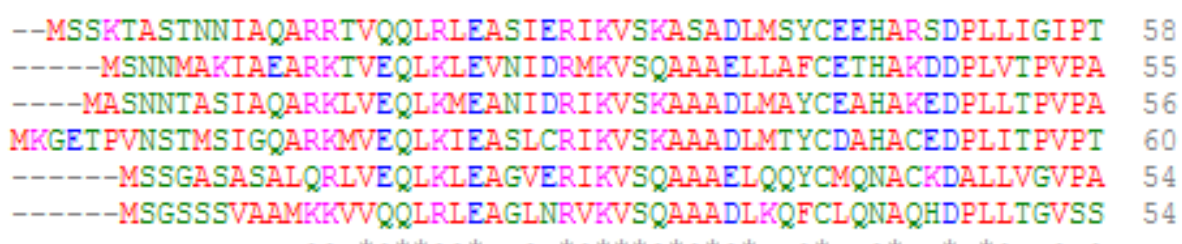

Fig 6. Primary sequence alignment of $G \gamma_{2}$-like subunits.

This study, and previous studies produced by our group, show an importance of positivelycharged basic arginine and lysine residues on both G $\gamma$ and SNAP25 (Wells et al, 2012). Despite this, a predominately positively-charged binding surface of both effectors would reasonably be anticipated to be repulsive. The identity of the negatively charged residues required for the G $\beta \gamma$ SNARE interaction is less well-understood. Two such G $\beta \gamma$-binding residues on SNAP25 are E62, located within the first of two helices SNAP25 contributes to the four-helix bundle, and D99, located within the flexible linker region connecting the first and second helices (Wells et al, 2012). The lack of secondary structure in the region where D99 is located would enable the residue to interface with a series of positively-charged residues identified in this data, including $G \gamma_{2} R 13$ and K14. However, since the positively charged K102 on SNAP25 is also a key binding residue in close proximity to D99, it would be predicted that a negatively charged residue would have to be in close proximity to R13 and K14, under this hypothesis. Three such residues exist in close 
proximity on the G $\alpha$-binding surface of $G \beta$, including D163, D186, and D205. On G $\gamma_{2}$, residue E17 is also in close proximity, but the data contradicts this, as mutagenesis of E17 to Ala does not interfere with the binding of $\mathrm{G} \gamma_{2}$ 13-27 to t-SNARE. The limitations of the ResPep technique, including the limited secondary structure of the 15-mer peptides, render it unable to identify every residue of importance within a given protein-protein interaction.

Given this detailed information on residues on both G $\beta \gamma$ and SNAP25, the ROSETTA modeling provides an intriguing and testable hypothesis for the interaction. The two models are distinct, with different regions of the protein interacting for both components of the full complex. The first model exhibits a 1:1 stoichiometry with a parallel coiled-coil orientation. The model highlights the importance of the C-terminus of SNAP25, a critical binding region for the interaction (Gerachshenko et al, 2005, Yoon et al, 2007, Wells et al, 2012, Zurawski et al, 2016), as well as the N-terminus of G $\gamma$ (Fig. 2, Fig. 3), Zurawski et al, 2017), all of which were shown to be important in binding and functional studies. Critically, the model emphasizes a 1:1 stoichiometry, while binding studies between labeled G $\beta_{1} \gamma_{1}$ and SNAP25 support two sites of interaction, with one closer to the N-terminus of the second helix of SNAP25 and the other at the C-terminus. Mutation of C-terminal binding residues reduces, but does not abolish the interaction (Wells et al, 2012).

The second model places the propeller domain of G $\beta$ adjacent to the central region of the second SNAP-25 helix. Superposition of the model atop the synaptotagmin-1:ternary SNARE crystal structure (Nature. 2015 Sep 3;525(7567):62-7) demonstrates a steric clash at the primary interface, consistent with a model of orthosteric competition between G $\beta \gamma$ and synaptotagmin 1 $\mathrm{C} 2 \mathrm{AB}$ for binding to this region predicted from biochemical experiments (Blackmer et al, 2005, Yoon et al, 2007, Zurawski et al, 2017, Zurawski et al., 2019). The G $\beta \gamma$-SNARE interaction is 
blocked in cellular studies through the use of C-terminal peptides derived from GRK2 (Blackmer et al, 2001), for which a crystal structure of the full GRK2 in complex with G $\beta \gamma$ exists (Mol Pharmacol. 2011 Aug;80(2):294-303). While the binding region of GRK2 is not the same as the second model, steric overlap of ternary SNARE and the GRK2 peptide occurs. Neither model features any sites of interaction adjacent to residue W332 of G $\beta$, but binding studies show that the W332A mutation significantly increases the affinity of G $\beta \gamma$ for t-SNARE complexes consisting of syntaxin1A and SNAP25 (Zurawski et al, 2017), however this may be allosteric in nature.

\section{Conclusions}

The interaction of the G-protein $\beta \gamma$ dimer with the SNARE complex is an important regulatory mechanism inhibiting exocytosis. In this study, we probed the regions of G $\beta \gamma$ important for its binding to the SNARE complex. Systematic peptide screening revealed two regions, consistent across the family of five $\mathrm{G} \beta$ and twelve $\mathrm{G} \gamma$ proteins, that can bind to tSNARE. These two regions, the G $\beta \gamma$ coiled-coil domain and the G $\beta$ propeller region, are spatially separated and are unable to act upon the same SNARE. Alanine scanning found that positively charged residues, on both $\mathrm{G} \beta$ and $\mathrm{G} \gamma$, are important for governing this interaction. Computational docking allowed for the systematic peptide analysis to be evaluated in scope of the entire complex. Analysis of the modeling was in agreement with the peptide screening, there are two distinct binding modes, using two different regions on both G $\beta \gamma$ and SNARE. This may allow for the formation of a higher order complex in vivo. These data, and the models based on them, clearly provide a roadmap for further experiments probing the nature of the interface. Obviously, it would be of great interest to determine the crystal structure to gain a high-resolution view of the G $\beta \gamma$-SNARE interaction. 


\section{Materials and Methods}

Materials. Primary antibodies SNAP25 (SC-376713) was from Santa Cruz Biotech. HRPconjugated secondary antibodies (anti-rabbit: 5520-0337, anti-mouse: 55220-0341) were from Seracare (Milford, MA). Enhanced chemiluminescence substrate (34580) was from ThermoFisher (Waltham, MA).

tSNARE and SNAP25 expression and purification. tSNARE was expressed and purified as described (Zurawski et al., 2017a).

Peptide Array analysis and Far-western blot. Peptide array synthesis was performed using the ResPep SL peptide synthesizer (Intavis AG, Koeln, Germany) according to standard SPOT synthesis protocols (Frank, 2002; Yim et al., 2015b). Indicated protein peptides containing 15 amino acids were directly coupled to membranes via the C-terminus during synthesis. Dried membranes with peptides were soaked in $100 \%$ ethanol for 5 min then rehydrated in water (twice $5 \mathrm{~min}$ ). The membranes were blocked for $1 \mathrm{~h}$ in Tris-buffered saline (TBS) with 5\% milk and $0.05 \%$ Tween 20 (Sigma-Aldrich, St. Louis, MO) and washed 3 times (5 min each) in TBS with $0.05 \%$ Tween 20 (TBS-T). The membranes were incubated overnight at $4^{\circ} \mathrm{C}$ with $\mathrm{t}$-snare protein at a final concentration of $0.5 \mu \mathrm{M}$ in $20 \mathrm{mM}$ MOPS, pH 7.5 buffer containing $150 \mathrm{mM} \mathrm{NaCl}$ and $2 \mathrm{mM}$ TCEP. The next morning, membranes were washed 3 times ( 5 min each) in TBS-T buffer and incubated with primary antibody (SNAP-25) at 1:1,000 dilution in TBS-T for $1 \mathrm{~h}$. Spots were detected using HRP-conjugated secondary antibodies as described by the manufacturer. For densitometric analysis, the signal in individual dots was quantified as a percentage of total density detected on the membrane (Quantity One and Image Lab, Bio Rad (Hercules, CA), Image J (Schneider et al., 2012)). This allowed for comparison of intensities across all peptide experiments. Statistical analysis was performed using one-way ANOVA followed by Dunnett's post-hoc test using GraphPad Prism 8.0.

Alphascreen competition-binding assays. Alphascreen luminescence measurements were performed as described (Zurawski et al., 2017a). Briefly, biotinylated SNAP-25 was diluted to a 5 concentration of $100 \mathrm{nM}$ in assay buffer (20 mM HEPES, pH 7.0, $10 \mathrm{mM} \mathrm{NaCl}, 40 \mathrm{mM} \mathrm{KCl}, 5 \%$ glycerol, and $0.01 \%$ Triton X-100). An EC80 concentration of $180 \mathrm{nM}$ purified His6-G $\beta_{1} \gamma_{2}$ was made in assay buffer. Peptide stocks in DMSO were spotted onto 384- well white OptiPlates (PerkinElmer Life Sciences) at concentration ranges of $1 \mathrm{nM}$ to $100 \mathrm{M}$ using a Labcyte Echo 555 Omics acoustic liquid handler (Labcyte, Sunnyvale, CA), with DMSO being back-added to a final concentration of $0.1 \%$. 4 ul of $\mathrm{G}_{1} \gamma_{2}$ solution was incubated with peptide for 5 min while shaking. After $5 \mathrm{~min}, 1 \mathrm{ul}$ of biotinylated SNAP25 was added to a final concentration of $20 \mathrm{nM}$. Subsequent to incubation while shaking for an additional $5 \mathrm{~min}, 10 \mathrm{ul}$ of Alphascreen Histidine Detection Kit (nickel chelate) acceptor beads were added to a final concentration of $20 \mathrm{ug} / \mathrm{ml}$ in assay buffer. The assay plate was agitated in dim green light for $30 \mathrm{~min}$. At that point, Alphascreen Streptavidin Donor Beads were added to a final concentration of $20 \mathrm{ug} / \mathrm{ml}$ in dim green light. All aqueous solutions in this assay were manipulated by a Velocity 11 Bravo liquid handler (Agilent Automation Solutions, Santa Clara, CA). The final volume in the assay plate was $25 \mathrm{ul}$. After being spun down briefly to settle all fluid at the bottom of the well, plates were incubated for an 
additional $1 \mathrm{~h}$ at $27{ }^{\circ} \mathrm{C}$ before being read in the EnSpire. $20 \mathrm{nM}$ biotinylated recombinant glutathione S-transferase in place of SNAP-25 with G $\beta_{1} \gamma_{2}$ were used as a negative control for nonspecific binding in each assay. IC50 concentrations for each peptide were determined by sigmoidal dose-response curve-fitting with variable slope. To have a strong signal in the Alphascreen competition-binding assay that could still be competed with a peptide, we used an EC80 concentration $(180 \mathrm{nM})$ of His6-tagged $\mathrm{G} \beta_{1} \gamma_{2}$ combined with $20 \mathrm{nM}$ recombinant human SNAP25 biotinylated on primary amine residues with NHS-biotin and increasing concentrations of peptides corresponding to a region on $\mathrm{G} \gamma_{2}$ in the Alphascreen bead assay. As a negative control, peptides were tested for their ability to disrupt a second Alphascreen assay in which donor and acceptor beads were reacted with $50 \mathrm{nM}$ concentrations of a peptide consisting of a biotinylation site and a His-tag peptide (PerkinElmer Life Sciences).

Computational docking. Using ROSETTA we applied the previously published protocols ROSETTADOCK to computationally dock the crystal structures of SNARE and G $\beta_{1} \gamma_{2}$. The experimentally determined SNARE structure was mutated at nine positions, from isoform 2 to isoform 1, to match the peptide screen sequence. Both structures were relaxed independently prior to docking. Rigid-body docking with sidechain flexibility was carried out at four distinct positions: placing the $\mathrm{G} \beta_{1} \gamma_{2}$ coiled-coil region parallel and anti-parallel to the $\mathrm{C}$-terminal region of SNAP-25, and placing the $G \beta^{82-96}$ and $G \beta^{124-138}$ of the propeller region located adjacent to SNAP25 residues 151-164 in two distinct orientations, with the $G \beta_{1}$ propeller orientation rotated 180 degrees. Approximately 2000 models were generated for each position. The generated models were evaluated by total complex energy and interface energy between the two binding partners using the REF2015 score function. The interface energy was calculated by determining the difference between the score of the bound complex versus the score of the separated complex with the side chains allowed to repack.

\section{References}

Bender, B. J., Cisneros, A., 3rd, Duran, A. M., Finn, J. A., Fu, D., Lokits, A. D., Mueller, B. K., Sangha, A. K., Sauer, M. F., Sevy, A. M., et al. (2016). Protocols for Molecular Modeling with Rosetta3 and RosettaScripts. Biochemistry 55, 4748-4763.

Betke, K. M., Wells, C. A., and Hamm, H. E. (2012). GPCR mediated regulation of synaptic transmission. Progress in neurobiology 96, 304-321.

Betty, M., Harnish, S. W., Rhodes, K. J., and Cockett, M. I. (1998). Distribution of heterotrimeric G-protein beta and gamma subunits in the rat brain. Neuroscience 85, 475-486.

Binz, T., Blasi, J., Yamasaki, S., Baumeister, A., Link, E., Sudhof, T. C., Jahn, R., and Niemann, H. (1994). Proteolysis of SNAP-25 by types E and A botulinal neurotoxins. The Journal of biological chemistry 269, 1617-1620.

Blackmer, T., Larsen, E. C., Bartleson, C., Kowalchyk, J. A., Yoon, E. J., Preininger, A. M., Alford, S., Hamm, H. E., and Martin, T. F. (2005). G protein betagamma directly regulates SNARE protein fusion machinery for secretory granule exocytosis. Nature neuroscience 8, 421-425.

Blackmer, T., Larsen, E. C., Takahashi, M., Martin, T. F., Alford, S., and Hamm, H. E. (2001). G protein betagamma subunit-mediated presynaptic inhibition: regulation of exocytotic fusion downstream of Ca2+ entry. Science 292, 293-297. 
Chaudhury, S., Berrondo, M., Weitzner, B. D., Muthu, P., Bergman, H., and Gray, J. J. (2011). Benchmarking and analysis of protein docking performance in Rosetta v3.2. PLoS One 6, e22477. Chen, Q., Iverson, T. M., and Gurevich, V. V. (2018). Structural Basis of Arrestin-Dependent Signal Transduction. Trends Biochem Sci 43, 412-423.

Chen, Q., Perry, N. A., Vishnivetskiy, S. A., Berndt, S., Gilbert, N. C., Zhuo, Y., Singh, P. K., Tholen, J., Ohi, M. D., Gurevich, E. V., et al. (2017). Structural basis of arrestin-3 activation and signaling. Nat Commun 8, 1427.

Chen, X., Tomchick, D., Kovrigin, E., Arac, D., Machius, M., Sudhof, T., and Rizo, J. (2002). Three-Dimensional Structure of the Complexin/SNARE Complex. Neuron 33, 397-409.

Clapham, D. E., and Neer, E. J. (1997). G protein beta gamma subunits. Annual review of pharmacology and toxicology 37, 167-203.

Clarke, S. (1992). Protein isoprenylation and methylation at carboxyl-terminal cysteine residues. Annual review of biochemistry 61, 355-386.

Cox, A. D. (1995). Mutation and analysis of prenylation signal sequences. Methods in enzymology 250, 105-121.

Currie, K. P. (2010). Inhibition of Ca2+ channels and adrenal catecholamine release by G protein coupled receptors. Cellular and molecular neurobiology 30, 1201-1208.

Delaney, A. J., Crane, J. W., and Sah, P. (2007). Noradrenaline Modulates Transmission at a Central Synapse by a Presynaptic Mechanism. Neuron 56, 880-892.

Downes, G. B., and Gautam, N. (1999). The G protein subunit gene families. Genomics 62, 544552.

Ernst, J. A., and Brunger, A. T. (2003). High resolution structure, stability, and synaptotagmin binding of a truncated neuronal SNARE complex. J Biol Chem 278, 8630-8636.

Ford, C. E., Skiba, N. P., Bae, H., Daaka, Y., Reuveny, E., Shekter, L. R., Rosal, R., Weng, G., Yang, C. S., Iyengar, R., et al. (1998). Molecular basis for interactions of G protein betagamma subunits with effectors. Science 280, 1271-1274.

Frank, R. (2002). The SPOT-synthesis technique. Synthetic peptide arrays on membrane supports-principles and applications. J Immunol Methods 267, 13-26.

Gautam, N., Downes, G. B., Yan, K., and Kisselev, O. (1998). The G-protein betagamma complex. Cell Signal 10, 447-455.

Gerachshenko, T., Blackmer, T., Yoon, E. J., Bartleson, C., Hamm, H. E., and Alford, S. (2005). Gbetagamma acts at the $\mathrm{C}$ terminus of SNAP-25 to mediate presynaptic inhibition. Nature neuroscience 8, 597-605.

Gray, J. J., Moughon, S., Wang, C., Schueler-Furman, O., Kuhlman, B., Rohl, C., and Baker, D. (2003). Protein-Protein Docking with Simultaneous Optimization of Rigid-body Displacement and Side-chain Conformations. Journal of Molecular Biology 331, 281-299.

Hamm, H. E., Deretic, D., Arendt, A., Hargrave, P. A., Koenig, B., and Hofmann, K. P. (1988). Site of $\mathrm{G}$ protein binding to rhodopsin mapped with synthetic peptides from the alpha subunit. Science 241, 832-835.

Herlitze, S., D.E. Garcia, K. Mackie, B. Hille, T. Scheuer, and W.A. Catterall (1996). Modulation of Ca2+ channels by G-protein bg subunits. Nature 380, 258-262.

Herlitze, S., Garcia, D. E., Mackie, K., Hille, B., Scheuer, T., and Catterall, W. A. (1996). Modulation of Ca2+ channels by G-protein beta gamma subunits. Nature 380, 258-262.

Hildebrandt, J. D. (1997). Role of subunit diversity in signaling by heterotrimeric G proteins. Biochemical Pharmacology 54, 325-339. 
Huang, C. L., Slesinger, P. A., Casey, P. J., Jan, Y. N., and Jan, L. Y. (1995). Evidence that direct binding of $\mathrm{G}$ beta gamma to the GIRK1 $\mathrm{G}$ protein-gated inwardly rectifying $\mathrm{K}+$ channel is important for channel activation. Neuron 15, 1133-1143.

Huang, X., Sun, S., Wang, X., Fan, F., Zhou, Q., Lu, S., Cao, Y., Wang, Q.-W., Dong, M.-Q., Yao, J., and Sui, S.-F. (2019). Mechanistic insights into the SNARE complex disassembly. Science Advances 5, 1-11.

Ikeda, S. R. (1996). Voltage-dependent modulation of N-type calcium channels by G-protein bg subunits. Nature 380, 255-258.

Lambright, D., Sondek, J., Bohm, A., Skiba, N., Hamm, H., and Sigler, P. (1996). The 2.0 A crystal structure of a heterotrimeric $\mathrm{G}$ protein. Nature 379, 311-319.

Lambright, D. G., Noel, J. P., Hamm, H. E., and Sigler, P. B. (1994). Structural determinants for activation of the alpha-subunit of a heterotrimeric G protein. Nature 369, 621-628.

Logothetis, D. E., Kurachi, Y., Galper, J., Neer, E. J., and Clapham, D. E. (1987). The betagamma subunits of GTP-binding proteins activate the muscarinic K+ channel in heart. Nature 325, 321326.

Maeda, S., Koehl, A., Matile, H., Hu, H., Hilger, D., Schertler, G. F. X., Manglik, A., Skiniotis, G., Dawson, R. J. P., and Kobilka, B. K. (2018). Development of an antibody fragment that stabilizes GPCR/G-protein complexes. Nat Commun 9, 3712.

Myung, C. S., Lim, W. K., DeFilippo, J. M., Yasuda, H., Neubig, R. R., and Garrison, J. C. (2006). Regions in the $\mathrm{G}$ protein gamma subunit important for interaction with receptors and effectors. Molecular pharmacology 69, 877-887.

Noel, J. P., Hamm, H. E., and Sigler, P. B. (1993). The 2.2 A crystal structure of transducin-alpha complexed with GTP gamma S. Nature 366, 654-663.

Panchenko, M. P., Saxena, K., Li, Y., Charnecki, S., Sternweis, P. M., Smith, T. F., Gilman, A. G., Kozasa, T., and Neer, E. J. (1998). Sites important for PLCbeta2 activation by the G protein betagamma subunit map to the sides of the beta propeller structure. The Journal of biological chemistry 273, 28298-28304.

Peng, L., Mirshahi, T., Zhang, H., Hirsch, J. P., and Logothetis, D. E. (2003). Critical determinants of the $\mathrm{G}$ protein gamma subunits in the Gbetagamma stimulation of $\mathrm{G}$ protein-activated inwardly rectifying potassium (GIRK) channel activity. The Journal of biological chemistry 278, 5020350211.

Photowala, H., Blackmer, T., Schwartz, E., Hamm, H. E., and Alford, S. (2006). G protein betagamma-subunits activated by serotonin mediate presynaptic inhibition by regulating vesicle fusion properties. Proceedings of the National Academy of Sciences of the United States of America 103, 4281-4286.

Reuveny, E., Slesinger, P. A., Inglese, J., Morales, J. M., Iniguez-Lluhi, J. A., Lefkowitz, R. J., Bourne, H. R., Jan, Y. N., and Jan, L. Y. (1994). Activation of the cloned muscarinic potassium channel by $\mathrm{G}$ protein beta gamma subunits. Nature 370, 143-146.

Sadja, R., and Reuveny, E. (2009). Activation gating kinetics of GIRK channels are mediated by cytoplasmic residues adjacent to transmembrane domains. Channels 3, 205-214.

Schiavo, G., Santucci, A., Dasgupta, B. R., Mehta, P. P., Jontes, J., Benfenati, F., Wilson, M. C., and Montecucco, C. (1993). Botulinum neurotoxins serotypes A and E cleave SNAP-25 at distinct COOH-terminal peptide bonds. FEBS letters 335, 99-103.

Schneider, C. A., Rasband, W. S., and Eliceiri, K. W. (2012). NIH Image to ImageJ: 25 years of image analysis. Nat Methods 9, 671-675. 
Simon, M. I., Strathmann, M. P., and Gautam, N. (1991). Diversity of G proteins in signal transduction. Science 252, 802-808.

Smrcka, A. V. (2008). G protein betagamma subunits: central mediators of G protein-coupled receptor signaling. Cellular and molecular life sciences : CMLS 65, 2191-2214.

Sondek, J., Bohm, A., Lambright, D., Hamm, H., and Sigler, P. (1996a). Crystal structure of a GA protein $\beta \gamma$ dimer at 2.1 $\AA$ resolution. Nature 379, 369-374.

Sondek, J., Bohm, A., Lambright, D. G., Hamm, H. E., and Sigler, P. B. (1996b). Crystal structure of a G-protein beta gamma dimer at 2.1A resolution. Nature 379, 369-374.

Wall, M. A., Coleman, D. E., Lee, E., Iniguez-Lluhi, J. A., Posner, B. A., Gilman, A. G., and Sprang, S. R. (1995). The structure of the G protein heterotrimer Gi alpha 1 beta 1 gamma 2. Cell 83, 1047-1058.

Wells, C. A., Zurawski, Z., Betke, K. M., Yim, Y. Y., Hyde, K., Rodriguez, S., Alford, S., and Hamm, H. E. (2012a). Gbetagamma inhibits exocytosis via interaction with critical residues on soluble N-ethylmaleimide-sensitive factor attachment protein-25. Molecular pharmacology 82, 1136-1149.

Wells, C. A., Zurawski, Z., Betke, K. M., Yim, Y. Y., Hyde, K., Rodriguez, S., Alford, S., and Hamm, H. E. (2012b). G $\beta \gamma$ Inhibits Exocytosis via Interaction with Critical Residues on Soluble N-Ethylmaleimide-Sensitive Factor Attachment Protein-25. Molecular pharmacology 82, 11361149.

Yim, Y. Y., Betke, K., and Hamm, H. (2015a). Using Peptide Arrays Created by the SPOT Method for Defining Protein-Protein Interactions. In Protein-Protein Interactions: Methods and Applications, C.L. Meyerkord, and H. Fu, eds. (New York, NY: Springer New York), pp. 307320.

Yim, Y. Y., Betke, K., and Hamm, H. (2015b). Using peptide arrays created by the SPOT method for defining protein-protein interactions. Methods Mol Biol 1278, 307-320.

Yim, Y. Y., Betke, K. M., McDonald, W. H., Gilsbach, R., Chen, Y., Hyde, K., Wang, Q., Hein, L., and Hamm, H. (2019). The in vivo specificity of synaptic G $\beta$ and G $\gamma$ subunits to the $\alpha 2 \mathrm{a}$ adrenergic receptor at CNS synapses. Scientific Reports 9, 1718.

Yim, Y. Y., McDonald, W. H., Betke, K.M., Kaya, A.I., Hyde, K., Gilsbach, R., Hein, L., Hamm, H.E. (2020). Specificity of Gbeta and gamma subunits to SNARE complex after $a_{2 a}$ adrenergic receptor stimulation. biorXiv.

Yoon, E. J., Gerachshenko, T., Spiegelberg, B. D., Alford, S., and Hamm, H. E. (2007). Gbetagamma interferes with Ca2+-dependent binding of synaptotagmin to the soluble Nethylmaleimide-sensitive factor attachment protein receptor (SNARE) complex. Molecular pharmacology 72, 1210-1219.

Yoon, E. J., Hamm, H. E., and Currie, K. P. (2008). G protein betagamma subunits modulate the number and nature of exocytotic fusion events in adrenal chromaffin cells independent of calcium entry. J Neurophysiol 100, 2929-2939.

Zhang, X.-1., Upreti, C., and Stanton, P. K. (2011). G $\beta \gamma$ and the C Terminus of SNAP-25 Are Necessary for Long-Term Depression of Transmitter Release. PLoS One 6, e20500.

Zhao, Q., Kawano, T., Nakata, H., Nakajima, Y., Nakajima, S., and Kozasa, T. (2003). Interaction of G Protein $\beta$ Subunit with Inward Rectifier K+ Channel Kir3. Molecular pharmacology 64, 10851091.

Zhao, Y., Fang, Q., Straub, S. G., Lindau, M., and Sharp, G. W. (2010). Noradrenaline inhibits exocytosis via the $\mathrm{G}$ protein betagamma subunit and refilling of the readily releasable granule pool via the alpha(i1/2) subunit. The Journal of physiology 588, 3485-3498. 
Zhou, Q., Lai, Y., Bacaj, T., Zhao, M., Lyubimov, A.Y., Uerirojnangkoorn, M., Zeldin, O.B., Brewster, A.S., Sauter, N.K., Cohen, A.E., Soltis, S.M., Alonso-Mori, R., Chollet, M., Lemke, H.T., Pfuetzner, R.A., Choi, U.B., Weis, W.I., Diao, J., Sudhof, T.C., Brunger, A.T. Nature 525, 62-67.

Zurawski, Z., Page, B., Chicka, M. C., Brindley, R. L., Wells, C. A., Preininger, A. M., Hyde, K., Gilbert, J. A., Cruz-Rodriguez, O., Currie, K. P. M., et al. (2017a). Gbetagamma directly modulates vesicle fusion by competing with synaptotagmin for binding to neuronal SNARE proteins embedded in membranes. The Journal of biological chemistry 292, 12165-12177.

Zurawski, Z., Page, B., Chicka, M. C., Brindley, R. L., Wells, C. A., Preininger, A. M., Hyde, K., Gilbert, J. A., Cruz-Rodriguez, O., Currie, K. P. M., et al. (2017b). G $\beta \gamma$ directly modulates vesicle fusion by competing with synaptotagmin for binding to neuronal SNARE proteins embedded in membranes. Journal of Biological Chemistry.

Zurawski, Z., Rodriguez, S., Hyde, K., Alford, S., and Hamm, H. E. (2016). G $\beta \gamma$ Binds to the Extreme C Terminus of SNAP25 to Mediate the Action of Gi/o-Coupled G Protein-Coupled Receptors. Molecular pharmacology 89, 75-83. 